\title{
》...THE ONLY THING WE'RE STRAIGHT ABOUT IS THE EDGE «1. DIE DISKUSSION ÜBER (HOMO-)SEXUALITÄT IN StRAight Edge
}

\author{
Merle Mulder
}

In der Öffentlichkeit wird immer wieder die zunehmende Verrohung der Jugend - vor allem im Hinblick auf Sexualität - beklagt. Zahlreiche Studien zeigen, dass >die heutige Jugend immer früher und immer mehr Sex zu haben scheint, und die Zahl der Teenagerschwangerschaften stetig steigt (vgl. z.B. Bundeszentrale für gesundheitliche Aufklärung 2002; 2006; 2009). Jenseits dieses gängigen Klischees des sexuell experimentierenden Jugendlichen hat sich innerhalb der kleinen, aber weltweit verbreiteten, im musikalischen Genre des Hardcore-Punk verorteten Gruppierung Straight Edge ein Verhältnis zu Sex und Sexualität etabliert, dass ganz und gar nicht diesem Bild entspricht. Unter dem Motto $\gg$ No Sex, No Drugs, Just Rock'n'Roll« werden nicht nur der Konsum von (legalen, wie illegalen) Rauschmitteln abgelehnt, positives Denken, »Open-Mindedness «, eine Do-ltYourself-Mentalität sowie Vegetarismus bzw. Veganismus gepriesen, sondern darüber hinaus auch die Ablehnung von Promiskuität, gegenseitiger Respekt und ein insgesamt verantwortungsvoller Umgang miteinander propagiert (vgl. Mulder 2009: 5-32). Ob all dies aber auch in der Umsetzung gelingt, welche unterschiedlichen Ansichten zu Sex und Sexualität sich innerhalb von Straight Edge finden lassen, welche Argumentationen hinter diesen stehen, wie sich rigide Auffassungen auch auf sexuelle Orientierungen auswirken, wie die Betroffenen sich dem entgegenstellen und welche Rolle die Musik dabei spielt, soll im Folgenden dargestellt werden.

1 Dan, Schlagzeuger der Gayrilla Biscuits, zit. n. \&amp 2002. 


\section{$\gg$ I am not anti-sex, I am anti-fucking «²: Straight Edge's Auffassungen zu Sex}

Bei Straight Edge wird, neben dem wohl wichtigsten Aspekt - der Zurückweisung von Rauschmitteln -, in der Regel auch Promiskuität, also der Sex mit häufig wechselnden Partnerinnen oder Partnern ohne feste Bindung, abgelehnt. ${ }^{3}$ Straight Edge entwickelte sich Anfang der 1980er Jahre in den USA. Zahlreiche Straight Edger der ersten Stunde berichten davon, dass sich zu diesem Zeitpunkt die Vorstellungen der »Hippies « von »freier Liebe« aus den 1960er und 1970er Jahren in vielen Bereichen der Gesellschaft etabliert hätten und die Grundlage für die »Yuppie-Exzesse« der 1980er Jahre bildeten (vgl. Azerrad 2001: 139ff.). Insbesondere Jugendliche fühlten sich unter Druck gesetzt, bereits in jungen Jahren sexuelle Erfahrungen gesammelt haben zu müssen (vgl. Haenfler 2006: 43). Gleichzeitig begannen die Massenmedien über das Thema HIV bzw. Aids zu berichten, das einen weiteren Schatten über die vermeintlichen Freuden eines unbedarften und ausschweifenden Sexuallebens warf (vgl. Hutter 1997: 86ff.).

Programmatische Funktion für Straight Edge hatte der Song »Out Of Step (With The World)« der US-amerikanischen Band Minor Threat von 1980, in dem Sänger lan Mackaye fordert: »Don't smoke, don't drink, don't fuck! « Das irritiert zunächst, denn wer diesen Aufruf wörtlich nimmt, könnte meinen, Straight Edger müssten völlig auf Sex verzichten, so wie sie es auch in Bezug auf das Rauchen und Trinken tun. Und in der Tat gibt es Anhängerinnen und Anhänger, die auch sexuell enthaltsam leben. Doch insgesamt ist das Thema umstritten und bildet die Grundlage für zahlreiche Machtkämpfe um die Definitionshoheit von Straight Edge. So finden sich die verschiedensten Ansichten: Von der vollständigen Enthaltsamkeit bis hin zu der Auffassung, dass Straight Edge und Sex nichts miteinander zu tun hätten.

Diejenigen, die davon ausgehen, dass Straight Edge und eine bestimmte Einstellung zu Sex nichts miteinander zu tun hätten, argumentieren meist, dass es für Straight Edge keine festgeschriebenen Regeln gäbe und vor allem die Songtexte von Minor Threat nicht als Regelwerk verstanden werden sollten, sondern es immer um eine persönliche Interpretation gehe (vgl. Haenfler 2006: 45). Auf diesen Aspekt wies auch Ideengeber MacKaye selbst mehr-

2 Der Sänger lan Mackaye, zit. n. Anderson/Jenkins 2001: 104.

3 An dieser Stelle sei darauf hingewiesen, dass die Begriffe »häufig « und »feste Bindung « je nach Betrachterin oder Betrachter variieren können und dies in der Regel auch tun. Das Konzept »Promiskuität« beruht somit auf einer äußerst vagen und damit nicht unproblematischen Definition. 
fach hin (vgl. Wood 2006: 71). Ähnlich äußern sich diejenigen Straight Edger, die Sex auch ohne feste Partnerin oder festen Partner für vertretbar halten, solange zuvor die gegenseitigen Erwartungen geklärt wurden. Sie beziehen sich weniger auf das »Don't fuck « als auf den Grundsatz des Respekts, welcher bei einer entsprechenden vorherigen Absprache gegeben sei.

Die vermutlich am weitesten verbreitete Auffassung unter Straight Edgern ist aber, dass Sex innerhalb einer längerfristigen festen Beziehung akzeptiert und Promiskuität im Gegenzug abgelehnt wird. Diese Einstellung geht vor allem auf Mackaye zurück, der den oben zitierten Vers entsprechend erklärt:

»Ich bin nicht gegen Sex, bin aber gegen das Ficken, wenn diese Unterscheidung verständlich ist. Diese ganze Scheiße, die ssexuell experimentierenden Jugendlichen ‘, sind zwiespältig. Im Fernsehen hat jeder Sex, doch niemand wird dabei schwanger oder bekommt eine Geschlechtskrankheit. Die Kids bekommen jedoch Probleme [wenn sie willkürlich Sex haben]« (MacKaye, zit. n. Andersen/Jenkins 2006: 113).

Sex wird hier also nicht grundsätzlich abgelehnt, sondern lediglich Promiskuität, da sie »destruktive« Tendenzen aufweise (vgl. Haenfler 2006: 44ff., 111; Maybaum 2003: 303f.).

Die Auffassung, Sex sei nur innerhalb der Ehe annehmbar, findet sich häufig bei Straight Edgern, für die der (insbesondere christliche) Glaube eine große Rolle spielt oder die - unabhängig von jeglichen religiösen Auffassungen - der Meinung sind, Sex sollte man sich für den Partner oder die Partnerin aufheben, mit der oder dem man den Rest seines Lebens verbringen möchte, was z.B. durch die Ehe gekennzeichnet sei (vgl. Haenfler 2006: 45; Maybaum 2003: 303f.; Wood 2006: 72). Noch einen Schritt weiter gehen Straight Edger mit der Einstellung, Sex sei nur zu Fortpflanzungszwecken akzeptabel. Diese Auffassung vertreten zum einen manche Hardliner, die seit den 1990er Jahren die bis dato eher liberale Auslegung der Straight Edge-Prinzipien immer rigider vertreten (vgl. Mulder 2009: 34ff.) und entsprechend nicht nur Sex ausschließlich zur Fortpflanzung akzeptieren, sondern auch Verhütungsmittel und Abtreibung strikt ablehnen (vgl. Schwarz 2005: 129). Diese Position findet sich ebenfalls oft in Kombination mit dem Hare Krishna-Glauben wieder, den einige Anhängerinnen und Anhänger in Straight Edge integriert haben (vgl. Mulder 2009: 47f.). Hier wird davon ausgegangen, dass Sex meist zu Ausbeutung, Manipulation, Respektlosigkeit und Krankheiten führe und eine Verschwendung von Lebensenergie sei (vgl. Langstone 2009; Cappo 1993: 51). Hare Krishna-Anhängern wird deshalb das Zölibat empfohlen. Die einzige Ausnahme bildet Sex innerhalb 
der Ehe, welcher der Fortpflanzung diene, denn dieser Sex sei laut Bhagavad-Gita - den Schriften der Hare Krishna - »religiös « (vgl. Cappo 1993: 50, 55ff.). Die Krishna Core-Band 108 verdeutlicht diese Einstellung z.B. in ihrem Song »Thorn $\ll$ :

»Chew the thorn, drink the blood, call it pleasure

But you'll never quench that thirst

Sex is suffering,

Bleed the envy, bleed the jealousy, bleed the heartache

Call it pleasure, chew the thorn. I won't«

(108: »Thorn«, 1994).

Sex wird hier nur als vermeintliches Vergnügen verstanden, welches vielmehr Leiden, Neid, Eifersucht und Schmerz zur Folge habe.

Die vermutlich konsequenteste Umsetzung des »Don't fuck « findet sich bei Straight Edgern jedoch in der vollständigen Enthaltsamkeit wieder. Hier wird argumentiert, dass Sex ebenso abhängig machen könne wie Drogen und Alkohol und den klaren Verstand bzw. die Selbstkontrolle beeinträchtige (vgl. Haenfler 2006: 111). Der Wunsch nach einem freien, wachen und unabhängigen Geist führt damit schlussendlich zu völliger Enthaltsamkeit. Diese Auffassung findet sich allerdings, ebenso wie Sex nur zu Fortpflanzungszwecken, eher selten.

Die Ansichten, in welchem Rahmen Sex stattfinden sollte, sind innerhalb von Straight Edge also nicht nur breit gestreut, sondern auch höchst umstritten, wobei an dieser Stelle auf globale und regionale Unterschiede hingewiesen sei und z.B. von einer strengeren Auslegung in den USA als in Europa auszugehen ist (vgl. Maybaum 2003: 304).

\section{»I Love Hardcore Boys, I Love Boys Hardcore ${ }^{4}$ : Gay Edge}

Die sehr strikten Sichtweisen innerhalb von Straight Edge gehen oft einher mit einer ebenfalls rigiden Auffassung zur sexuellen Orientierung. So finden sich z.B. bei Hardlinern, Krishna Straight Edgern oder streng gläubigen Christen teils auch homophobe Ansichten wieder. Vor allem Hardliner berufen sich in dieser Hinsicht auf das Prinzip der »Natural Order « (vgl. Peterson 2009: 93, 481ff.), das Sean Muttaqi, Hardline-Begründer und Sänger der Band Vegan Reich, folgendermaßen verteidigt:

4 Limp Wrist-Song auf: Complete Discography, 2004. 
»In regards to what some people perceived as >homophobia< was the stand hardline took that said if you were hardline, you could not be gay, that homosexuality was not part of a healthy, natural lifestyle from its perspective. [...] I always thought it was funny that Hardliners would get flak for simply deciding that homosexuality was a lifestyle they didn't want to engage in or stating they believed it was wrong (Muttaqi, zit. n. Peterson 2009: 96f.).

Entsprechende Anhänger sind innerhalb von Straight Edge anteilsmäßig zwar in der klaren Unterzahl, aber durch ihr meist sehr bestimmtes, teilweise sogar militantes Auftreten machen sie sich umso stärker bemerkbar.

Auch jenseits dieser militanten oder religiös motivierten Ablehnung berichten homosexuelle Straight Edger von einer »testosteronerfüllten« und von hetero-sexistischen, hypermaskulinen »tough guys « durchzogenen Straight Edge- und Hardcore-Szene sowie einer allgemeinen Unbedarftheit im Umgang mit Schimpfwörtern wie »Schwuchtel« oder Aussagen wie »Das ist doch voll schwul« (Wauz 2009). Bruce LaBruce, kanadischer Underground-Filmemacher und Fotograf, beschreibt die Situation wie folgt: »[B]y the mid '80s, with the advent of hardcore and the mosh pit, a new era of machismo and heterosexual rigidity was ushered in« (LaBruce, zit. n. Ciminelli/Knox 2005: 8). Dies zeigte sich z.B. daran, dass Homosexuelle vor allem beim >kontaktstarken< Slam-Dancen auf Konzerten von Heterosexuellen teilweise als »Bedrohung « wahrgenommen wurden, wie Sean Capone, Macher des »queeren« Straight Edge-Fanzines Positron, berichtet: »A queer in the pit was a threat to the normative sublimation of hetero-male aggression that can only come from physical contact with another man« (Capone, zit. n. Peterson 2009: 39).

So fühlen sich viele homosexuelle Straight Edger von den heterosexuellen ausgeschlossen oder zumindest nicht akzeptiert, obwohl sie sich denselben Regeln verschrieben haben und dieselbe Musik lieben (vgl. \&amp 2002; Anon. 2008). Dies führte seit Mitte der 1990er Jahre dazu, dass sich in den USA einige sogenannte »Gay Edge «-Bands wie die Gayrilla Biscuits, Youth of Togay oder Limp Wrist gründeten, die offensiv und lautstark auf die Diskriminierung Homosexueller in den eigenen Reihen aufmerksam machen. Ihr Ziel ist es, ein Klima der Sicherheit zu schaffen, in dem sich Homosexuelle ohne Vorbehalte outen können und ihre Sexualität nicht mehr zu verstecken brauchen, sondern sie stolz nach außen tragen können (vgl. Ciminelli/Knox 2005: 6; Anon. 2008; Gay Edge Liberation 2007).

In Deutschland gibt es bislang keine Gay Edge-Bands, doch auch hier ist das Thema aktuell. So hat sich 2007 das Gay Edge Liberation-Projekt gegründet, das in der Szene ebenfalls versucht auf das Thema Homophobie in Straight Edge und Hardcore aufmerksam zu machen. Hierfür haben die Pro- 
jektgründer den Rotten Schwuchtel-Sampler herausgegeben, der bereits veröffentlichte Songs von zahlreichen deutschen und internationalen Punkund Hardcore-Bands wie Black Fag, Oi Polloi, WIZO, MDC und Kafkas beinhaltet, die sich kritisch mit dem Thema Homophobie auseinandersetzen (vgl. Gay Edge Liberation 2007; Wauz 2009).

Wie im Folgenden noch zu sehen sein wird, zeigt sich in Band- und Künstlernamen, der Covergestaltung der Platten, der Songtitel-Wahl und einem Großteil der Songtexte der Gay Edger, dass sie sehr explizit, sehr sexuell und provokativ sind. Auf den erfahrenen Heterosexismus bzw. die vorherrschende Heteronormativität in der Gesellschaft, aber auch der eigenen Szene, scheint dabei mit einer Art performativer Hyperhomosexualität reagiert zu werden. Hierfür bedienen sie sich zweier spezieller (Stil-)Mittel - der Parodie und dem Pastiche - mit Hilfe derer die eigenen Anliegen auf die Agenda gesetzt werden.

Parodie wird hier verstanden als Mittel, bei dem die Eigenheiten eines bestimmten Stils imitiert und dabei das Original verspottet wird, jedoch auf eine Art und Weise, die stets eine gewisse Sympathie mit dem Original voraussetzt (vgl. Jameson 1983: 113). Ihre Verwendung soll am Beispiel der Gay Edge-Band Youth of Togay verdeutlicht werden und zeigt sich bereits bei deren Namensgebung, für die die Straight Edge-Größe Youth of Today Pate stand. Diesem Humor folgen die Bandmitglieder auch im Hinblick auf die eigenen (Künstler-)Namen: Sänger Gay Cappo benennt sich nach Ray Cappo (Sänger von Youth of Today), Gitarrist Mike Fudge nach Mike Judge (Sänger der Band Judge) usw. Ebenso sind viele Songs der Gay Edge-Band Parodien bekannter Straight Edge-Hymen. So interpretierten Youth of Togay z.B. den Song »Straight Edge Revenge« von Project X (1987) um in »Gay Edge Revenge« (2006), aus Slapshots »Last Laugh« (2003) wurde »Lust Laugh« (2008). Die Band schreibt darüber hinaus eigene Songs wie »Dude Of Your Dreams « (2008) oder »Adam And Steve« (2006). Und auch die Plattencover bekannter Straight Edge- und Hardcore-Bands sind nicht vor der Gay Edge-Band sicher. So wurde, wie in Abbildung $1 \mathrm{zu}$ sehen, das Cover der Can't Close My Eyes-EP von Youth of Today (1985), auf dem Sänger Ray Cappo in ein Mikrophon schreiend, der Handrücken mit dem X - dem >Erkennungszeichen ‘ für Straight Edge - verziert, zu sehen ist, von Youth of Togay für das Cover ihrer Platte Want More Guys (2006) bearbeitet, indem sie das Mikrophon durch einen Penis ersetzten und Sperma von der Wange Ray Cappos tropfen lassen, das X ersetzt durch den Winkel in den Farben der Regenbogenflagge. ${ }^{5}$ Auf ähnliche Art und Weise bearbeitete die Band

5 Anlehnung an den »Rosa Winkel« (»pink triangle«) - ein auf den Kopf gestelltes, rosafarbenes Dreieck. Im Zweiten Weltkrieg benutzten die Nationalsozialis- 
z.B. auch das Cover des Albums The Things We Carry (2007) von Have Heart für ihre 7"-EP The Dongs We Bury (2007).
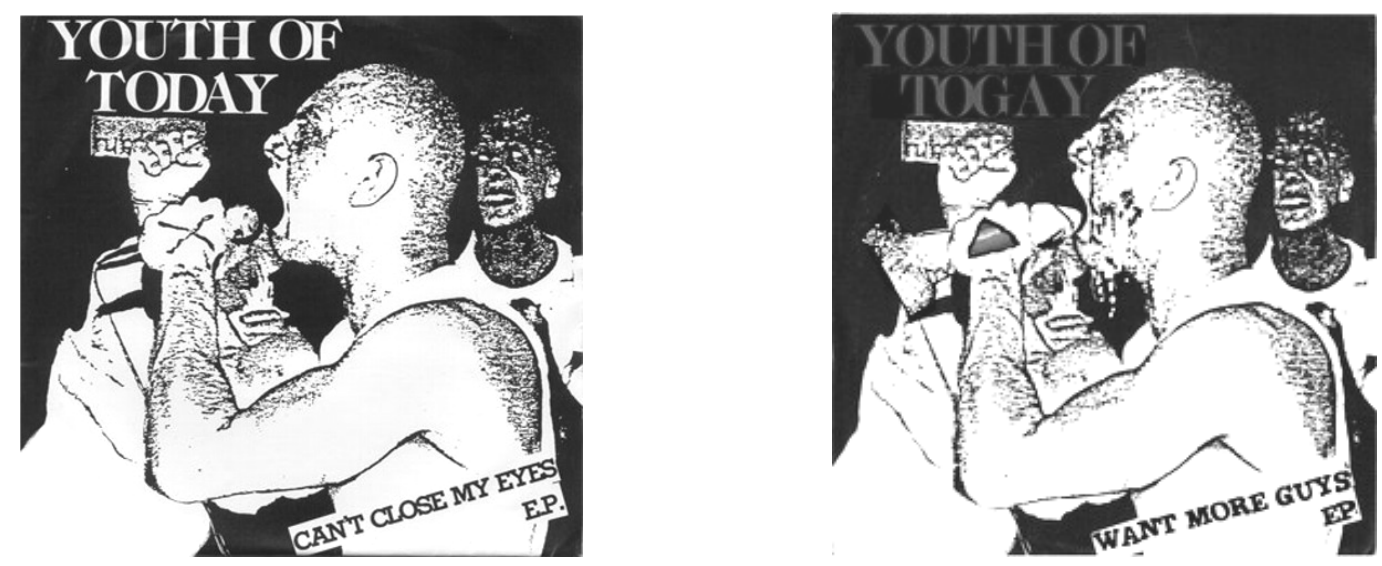

Abb. 1: Links: Plattencover Can't Close My Eyes (in der Originalversion von 1985) der Band Youth of Today. Rechts: Parodierendes Plattencover Want More Guys (2006) der Band Youth of Togay.

Schließlich folgen auch zahlreiche Songtexte von Youth of Togay diesem Parodie-Muster. Als Beispiel sei hier der Text zum Titel »Gay Edge Revenge« aufgeführt. Im Original »Straight Edge Revenge« von Project $X$ heißt es noch:

»I'm as straight as the line that you sniff up your nose

I'm as hard as the booze that you swill down your throat

I'm as bad as the shit you breath into your lungs

And I'll fuck you up as fast as the pill on your tongue

Straight Edge Revenge

This time you pushed me too far!«

(Project X: »Straight Edge Revenge«, 1987)

Youth of Togay machen daraus später:

$» I ' m$ as gay as the dude on the 3 Dollar Bill

I'm as yummy as the gizz that you put down your throat

I'm as hard as the cock that you put in my ass

And I'll fuck your ass as fast ... well fast

Gay Edge Revenge

This time you made me so hard

ten in den Konzentrationslagern, je nach Einlieferungsgrund, verschiedenfarbige Winkel, um die Häftlinge zu kennzeichnen. Menschen, die aufgrund ihrer Homosexualität bzw. eines Homosexualitätverdachts eingeliefert wurden, wurden mit dem Rosa Winkel gekennzeichnet. Jahre später wurde das ursprüngliche Stigma des Rosa Winkel von der Schwulenbewegung in ein positiv besetztes internationales Erkennungssymbol umgewandelt (vgl. Heger 1972: 32f.; Stümke 1989: 103, 127ff.; Stümke/Finkler 1981: 268ff.). 
I'm here and I'm queer and we won't go away

We suck balls, we fuck asses, because we're fucking gay

You think we're ..., well my friend you're fucking wrong

We're just like you except ... we love dong!«

(Youth of Togay: »Gay Edge Revenge«, 2008)

Aber nicht jeder Song, nicht jede Platte und nicht jeder Auftritt, welche/r auf den ersten Blick lustig erscheint, ist von Gay Edgern auch so gemeint. Dan, Schlagzeuger der Gay Edge-Band Gayrialla Biscuits erklärt:

"A lot of people think the songs are funny, and they are to most people. To me they are an example of our militant homosexual feelings. We are angry about our society that represses sexual expression. [...] The songs are funny, but they represent how we feel. Gay hardcore pride is here to stay! « (\&amp 2002).

Gerade vor diesem Hintergrund zeigt sich, dass der Begriff der Parodie in Zusammenhang mit Gay Edge nicht immer treffend ist. Vielmehr scheint hier das Konzept des Pastiche, wie es der amerikanische Literaturtheoretiker Fredric Jameson in seinem Aufsatz »Postmodernism and Consumer Society« entwickelte, als geeigneter:

»Pastiche is, like parody, the imitation of a peculiar or unique style, the wearing of a stylistic mask, speech in a dead language: but it is a neutral practice of such mimicry, without parody's ulterior motive, without the satirical impulse, without laughter, without that still latent feeling that there exists something normal compared to which what is being imitated is rather comic. Pastiche is blank parody, parody that has lost its sense of humor « (Jameson 1983: 114).

Innerhalb von Gay Edge fällt eine eindeutige Zuordnung jedoch nicht immer leicht. Folgt man der Interpretation der amerikanischen Literaturwissenschaftlerin Judith Butler von Jamesons Konzept »müßten wir die schwulen Identitäten eher als Pastiche verstehen «, denn

»[w]ährend die Parodie nach Jameson noch eine Sympathie mit dem Original bewahrt, dessen Kopie es ist, bestreitet das Pastiche die Möglichkeit eines >Originals< bzw. offenbart, was die Geschlechtsidentität betrifft, das >Original< als einen verfehlten Versuch, ein phantasmatisches Ideal zu kopieren, das nicht ohne Verfehlung kopiert werden kann« (Butler 1991: 226).

Nichts desto trotz scheint eine ausschließliche Zuordnung von Gay Edge zum Pastiche oder zur Parodie als zu eng; eher sind beide Möglichkeiten gegeben, wobei die Übergänge oftmals fließend sind. 
Das provokante Auftreten der Gay Edger hat zwei Ziele: Einerseits werden die weit verbreiteten Vorstellungen und Vorurteile von Heterosexuellen über Homosexuelle nicht nur bedient, sondern extrem überspitzt und damit zwar oftmals ins Komische gewandt, gleichzeitig jedoch auch angeprangert. Butler gibt für diese Art der Performanz einen interessanten Erklärungsversuch:

»Man betrachte nur die ent-machtenden und ent-naturalisierenden Effekte eines spezifisch schwulen Umgangs mit den heterosexuellen Konstrukten: Die Präsenz dieser Normen stellt nicht nur einen Schauplatz der Macht dar, den man nicht zurückweisen kann, sondern kann und wird zudem tatsächlich zum Schauplatz der parodistischen Anfechtung und Zurschaustellung, die der Zwangsheterosexualität ihren Anspruch auf Natürlichkeit und Ursprünglichkeit nimmt« (Butler 1991: 184).

Demnach könnte der Gay Edge-spezifische Umgang mit den Stilmitteln Parodie und Pastiche also als Versuch gewertet werden, die vorherrschende Heteronormativität innerhalb von Straight Edge und Hardcore umzustürzen und ein Machtgleichgewicht zwischen homo- und heterosexuellen Straight Edgern herzustellen. Eine solche Interpretation scheint im Falle von Gay Edge durchaus zulässig.

Andererseits richten sich Gay Edger mit ihrer performativen Hyperhomosexualität aber auch gegen den shomosexuellen Mainstream< und dessen promisken Lebensstil. ${ }^{6}$ Denn auch wenn es auf den ersten Blick vielleicht nicht deutlich wird: Gay Edger sind eben auch Straight Edger und lehnen Promiskuität für sich ab (vgl. \&amp 2002). Scotty Niemet, Herausgeber des »queeren« Let Me Live...-Fanzines, führt seine Ablehnung des schwulen Mainstreams dabei auch auf dessen Übernahme des Verhaltens und der Normen des >gesamtgesellschaftlichen< Mainstreams zurück:

»The gay community as a generalized whole bores me. It has created a culture with rules and mannerisms that mirror those of mainstream America and puts things such as wealth, career, and beauty as signs of success. I'm a punk kid and I will not compromise those values « (Niemet, zit. n. Peterson 2009: 40f.).

6 Ob dieser tatsächlich einen überwiegend promisken Lebensstil verfolgt, konnte bisher nicht endgültig geklärt werden. Das dies aber der Fall sei, behauptet zumindest der nicht unumstrittene Soziologe und Homosexualitätsforscher Rüdiger Lautmann: »Immerhin kann als sicher gelten, daß Homosexuelle in höherem Maße promisk sind als Heterosexuelle (was nicht zuletzt darauf beruht, daß sie kaum auf legitime Weise mit jemandem zusammenleben können)« (Lautmann 1984: 12). 
Unabhängig davon zeigt sich der zuvor betonte »schwule Stolz« u.a. darin, dass sich einige Gay Edger, ähnlich wie Straight Edger mit dem schwarzen X, mit einem schwarzen Winkel auf dem Handrücken selbst kennzeichnen und damit nicht nur ihre Straight Edge-, sondern auch ihre homosexuelle Identität öffentlich zur Schau tragen (zu sehen auf dem Cover der Gayrilla Biscuits in Abbildung 2). Inwiefern diese Form der Selbststigmatisierung jedoch auch wieder Parodie ist, ist dabei nicht immer deutlich auszumachen (so ist z.B. das Gayrilla Biscuits-Plattencover eine Anlehnung an das Plattencover der Band Project X).

\section{GAYRILLA BISCUITS}

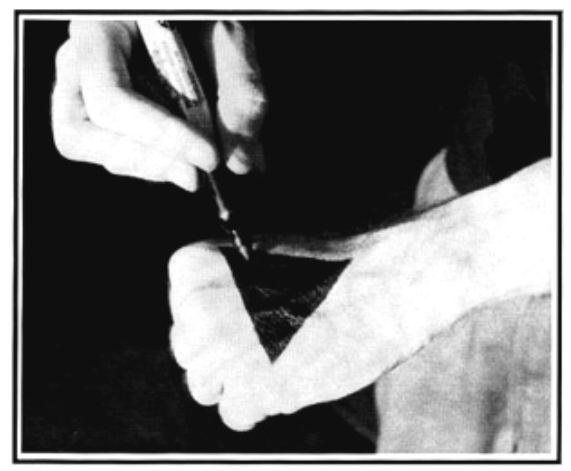

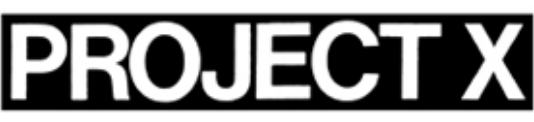

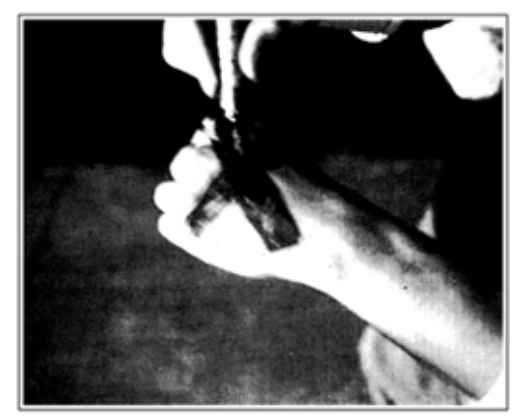

Abb. 2: Links: Plattencover The Demos (1998) mit dem "Schwarzen Winkel « der Band Gayrilla Biscuits. Rechts: Original-Plattencover Straight Edge Revenge (1987) der Band Project X.

Doch warum vor allem die Parodie als primäres Ausdrucksmittel? Youth of Togay liefern hierfür eine einleuchtende Erklärung: Sie weisen zunächst auf den Umstand hin, dass viele Menschen sich wohler fühlen, wenn sie über ein ernstes Thema lachen dürfen, anstatt mit erhobenem Zeigefinger belehrt zu werden. Dementsprechend ist es der ausdrückliche Wunsch der Band, dass die Zuhörer ihre Songs witzig finden - und dann vielleicht die »Message « dahinter verstehen. So nehme man zum Thema Homosexualität und Homophobie zwar einen humorvollen Standpunkt ein, die Einstellungen und Gefühle bezüglich dieser Themen seien aber genauso stark und genauso ernst gemeint, wie bei Künstlerinnen und Künstlern, die sich in einer »ernsthafteren« Weise ausdrückten. Des Weiteren erläutern Youth of Togay, dass sie mit ihren Songs zwar persönliche und auch schmerzvolle Erfahrungen einer Öffentlichkeit präsentierten, dabei jedoch trotzdem in der Lage seien, über diese Erlebnisse zu lachen, was dazu führe, dass sie sich selbst dabei wohler fühlen, wenn sie das Erlebte mit anderen teilen (vgl. Anon. 2008). 
Es sei jedoch noch anzumerken, dass sich nicht jeder homosexuelle Straight Edger unweigerlich als Gay Edge versteht. So stellt sich immer die Frage, welchen Stellenwert Sexualität und die sexuelle Orientierung bzw. Homosexualität nicht nur innerhalb der eigenen Musik, sondern vor allem auch innerhalb der eigenen Identität einnehmen. Dabei empfinden einige ein entsprechendes Label - sei es nun »Gay Edge«, »Queercore« oder »Homocore « - als einschränkend. Der frühere Sänger der Queercore-Band Extra Fancy, Brian Grillo, beschreibt dieses Gefühl folgendermaßen:

»I'm a little uncomfortable with this label >homocore $<$. [...] I guess it might be fine for some, but if you have ever listened to my music or seen my art, you might notice that there is a lot more than just the fact that I'm attracted to guys«(Grillo, zit. n. Ciminelli/Knox 2005: 44).

Unabhängig davon kommen Gay Edger schließlich zu dem Ergebnis, dass Wut, Zorn und Politik innerhalb von Straight Edge und Hardcore in jedem Falle ihren Platz haben, aber ebenso müsse Raum für Spaß und Humor vorhanden sein (vgl. Anon. 2008).

\section{»I think people have done good things with it. And some dumb shit, too ${ }^{7}$ : Fazit}

Auch eine Szene, die für sich selbst beansprucht, tolerant und aufgeschlossen zu sein, liefert ein breites Spektrum an Einstellungen zu Sex und Sexualität, die z.T. den Grundgedanken von Respekt und Open-Mindedness und auch der Selbsteinschätzung, aufgeklärter und fortschrittlicher als andere zu sein, widersprechen. So erscheint die Beurteilung von Norman Brannon, Gitarrist u.a. in der Krishnacore-Band Shelter und Autor des Anti-MatterFanzines, bezüglich des Einflusses des gesellschaftlichen Mainstreams auf Straight Edge und Hardcore recht treffend:

»Hardcore still is a white, male, heterosexual playground. Any way you cut it, it's the truth. In that sense, hardcore is still as progressive as it can be, but it's still a reflection of the mainstream society, which is run by white, heterosexual males. In that greater society there is such a long way to go with these issues, so the hardcore scene has similar problems. It might run on a faster track in hardcore, but it's still perhaps not as progressive as it would like to think it is« (Brannon, zit. n. Peterson 2009: 38).

Dies zeigt sich vor allem daran, dass auch Straight Edge die jahrhundertealten Machtkämpfe, mit denen sich unsere Gesellschaft in Bezug auf legi-

7 Ian MacKaye, zit. n. Blush 2001: 28. 
time Sexualität - seien es nun Praktiken oder Orientierungen - auseinandersetzt, bisher nicht überwinden konnte.

Abschließend sei jedoch auch noch einmal darauf hingewiesen - ohne die vorherigen Ausführungen relativieren zu wollen -, dass Straight Edger nicht grundsätzlich homophob sind. So stellt Brian Peterson, Autor des Buches Burning Fight. The Nineties Hardcore Revolution in Ethics, Politics, Spirit, and Sound, einen Wandel der Wahrnehmung und Einstellung bei Hardcore und Straight Edge gegenüber dem Thema Homosexualität fest:

»Things started to change in the nineties though as a variety of zines that dealt with homosexuality like Positron and Kill the Robot, as well as may prominent members of the scene coming out about their sexuality, helped make gay issues an important part of the dialogue during this era. Homophobia became less acceptable as the years passed « (Peterson 2009: 37).

Auch jenseits von Gay Edge kritisieren prominente Hetero-Straight EdgeBands wie z.B. Earth Crisis, Outspoken oder Good Clean Fun Homophobie und sprechen Homosexuellen ihre Unterstützung aus. Aber es gibt eben auch die andere Seite von Straight Edge und Hardcore. Und trotz dieser offensichtlich bestehenden Schwierigkeiten treten Gay Edger offensiv und kreativ für die Problematisierung von Vorurteilen und Diskriminierung der Homosexuellen ein und nutzen dabei insbesondere die Musik zur Thematisierung ihrer Interessen und Ziele.

\section{Literatur}

Eamp (2002). „Gayrilla Biscuits« [Interview]. In: Buddyhead, http://www. buddyhead.com/interview-with-gayrilla-biscuits, Version v. 31.5.2002 (Zugriff am 13.6.2010).

Andersen, Mark / Jenkins, Mark (2001). Dance of Days: Two Decades of Punk in the Nation's Capital. New York: Soft Skull Press.

Andersen, Mark / Jenkins, Mark (2006). Punk, DC. Dance of Days. Washington Hardcore von Minor Threat bis Bikini Kill. Mainz: Ventil.

Anon. (2008). „Got the Queer Edge? An Interview with Youth of Togay! « In: Left Of The Dial, http://www.leftofthedialmag.com/?p=431, Version v. 13.4.2008 (Zugriff am 13.6.2010).

Azerrad, Michael (2001). Our Band Could Be Your Life. Scenes from the American Indie Underground 1981-1991. New York, Boston, London: Little, Brown and Company.

Blush, Steven (2001). American Hardcore: A Tribal History. Los Angeles, New York: Feral House.

Breyvogel, Wilfried (Hg.) (2005). Eine Einführung in Jugendkulturen. Veganismus und Tattoos. Wiesbaden: VS Verlag für Sozialwissenschaften. 
Bundeszentrale für gesundheitliche Aufklärung (Hg.) (2002). Jugendsexualität: Wiederholungsbefragung von 14- bis 17-Jährigen und ihren Eltern. Ergebnisse der Repräsentativbefragung aus 2001. Köln: BZgA.

Bundeszentrale für gesundheitliche Aufklärung (Hg.) (2006). Jugendsexualität: Wiederholungsbefragung von 14- bis 17-Jährigen und ihren Eltern. Ergebnisse der Repräsentativbefragung aus 2005. Köln: BZgA.

Bundeszentrale für gesundheitliche Aufklärung (Hg.) (2009). Schwangerschaft und Schwangerschaftsabbruch bei minderjährigen Frauen. Köln: BZgA.

Butler, Judith (1991). Das Unbehagen der Geschlechter. Frankfurt/M.: Suhrkamp.

Cappo, Ray (1993). In Defense of Reality. Conversations between Ray Cappo \& Satyaraj Das. Hudson, NY: Equal Vision Records.

Ciminelli, David / Knox, Ken (2005). Homocore. The Loud and Raucous Rise of Queer Rock. Los Angeles: Alyson Books.

Foster, Hal (Hg.) (1983). The Anti-Aesthetic: Essays on Postmodernism Culture. Port Townsend: Bay Press.

Gay Edge Liberation (2007). MySpace-Seite, http://www.myspace.com/gayedge liberation (Zugriff am 13.6.2010).

Haenfler, Ross (2006). Straight Edge: Hardcore Punk, Clean-Living Youth, and Social Change. New Brunswick: Rutgers University Press.

Heger, Heinz (1972). Die Männer mit dem rosa Winkel. Hamburg: Merlin-Verlag.

Hutter, Jörg (1997). "Aids und die gesellschaftliche Moralpanik.« In: Vorgänge: Zeitschrift für Bürgerrechte und Politik 3, S. 86-95.

Jameson, Fredric (1983). »Postmodernism and Consumer Society.« In: The AntiAesthetic: Essays on Postmodernism Culture. Hg. v. Hal Foster. Port Townsend, WA: Bay Press, S. 111-125.

Langstone, Mark (2009). »Over the Edge.«In: xsisterhoodx.com. a straight edge and hardcore community, http://www.xsisterhoodx.com/straight-edge/x-overthe-edge-x.html, Version v. 26.1.2009 (Zugriff am 13.6.2010).

Lautmann, Rüdiger (1984). Seminar: Gesellschaft und Homosexualität. Frankfurt/M.: Suhrkamp (2. Aufl.).

Luig, Ute / Seebode, Jochen (Hg.) (2003). Ethnologie der Jugend: Soziale Praxis, moralische Diskurse und inszenierte Körperlichkeit. Münster, Hamburg, London: Lit.

Maybaum, Frederik (2003). »`... and let me live poison free . Zum Verhältnis von Jugendkultur und Reinheitsvorstellungen. Am Beispiel der Werte des straight edge. «In: Ethnologie der Jugend: Soziale Praxis, moralische Diskurse und inszenierte Körperlichkeit. Hg. v. Ute Luig und Jochen Seebode. Münster, Hamburg, London: Lit, S. 295-325.

Mulder, Merle (2009). Straight Edge: Subkultur, Ideologie, Lebensstil? Münster: Telos.

Peterson, Brian (2009). Burning Fight. The Nineties Hardcore Revolution in Ethics, Politics, Spirit, and Sound. Huntington Beach, CA: Revelations Records Publishing.

Schwarz, Thomas (2005). „Veganismus und das Recht der Tiere. Historische und theoretische Grundlagen sowie ausgewählte Fallstudien mit Tierrechtlern bzw. Veganern aus musikorientierten Jugendszenen. « In: Eine Einführung in Jugendkulturen. Veganismus und Tattoos. Hg. v. Wilfried Breyvogel. Wiesbaden: VS Verlag für Sozialwissenschaften, S. 69-163.

Stümke, Hans-Georg / Finkler, Rudi (1981). Rosa Winkel, Rosa Liste. Homosexuelle und "Gesundes Volksempfinden« von Auschwitz bis heute. Reinbek bei Hamburg: Rowohlt. 
Stümke, Hans-Georg (1989). Homosexuelle in Deutschland. Eine politische Geschichte. München: Beck.

Wauz (2002). »Gay Edge Liberation« [Interview]. In: Beatpunk Webzine, http:// www.beatpunk.org/interviews/gay-edge-liberation, Version v. 13.2.2009 (Zugriff am 13.6.2010).

Wood, Robert T. (2006). Straightedge Youth: Complexity and Contradictions of a Subculture. Syracuse, NY: Syracuse University Press.

\title{
Diskographie
}

108 (1994). Songs of Separation. LP. Equal Vision Records. Gayrilla Biscuits (1998). The Demos. EP. Slowgun Records. Have Heart (2007). The Things We Carry. LP. Bridge 9 Records. Limp Wrist (2004). Complete Discography. LP. Paralogy Records. Minor Threat (1981). Minor Threat. EP. Dischord.

Project X (1987). Straight Edge Revenge. EP. Schism Records. Slapshot (2003). Digital Warfare. LP. Bridge 9 Records. Youth of Today (1985). Can't Close My Eyes. EP. Positive Force Records. Youth of Togay (2006). Want More Guys. EP. FNS Records. Youth of Togay (2007). The Dongs We Bury. 7"-EP. FNS Records. Youth of Togay (2008). Tossed Salad Days. LP. FNS Records/Rejected Records.

\begin{abstract}
In the past 30 years followers of the music-orientated Straight Edge phenomenon have taken a stance on sexuality, which totally counteracts the current cliché of the »sexually experimenting adolescent «. But behind the slogan »No Sex, No Drugs, Just Rock'n'Roll« one can find numerous sentiments, competing for the authority to define legitimate sexuality concerning sexual practices and orientations. As a reaction to especially rigid sentiments the subgroup "Gay Edge emerged. With the help of some kind of performative hyper-homosexuality and the (stylistic) means of parody and pastiche they try to challenge the prevalent heteronormativity.
\end{abstract}

